\title{
Development of Correctors: From O. Scherzer to TEAM
}

\author{
M. Haider, P. Hartel, H. Müller, S. Uhlemann and J. Zach \\ CEOS GmbH, Englerstr. 28, D-69126 Heidelberg, Germany
}

The development of correctors started with the first paper on this subject by Scherzer in 1936 [1] in which he described the impossibility of designing an objective lens free of spherical and chromatic aberration. In 1947, Scherzer proposed three possibilities to compensate the spherical and chromatic aberration of a rotationally symmetric objective lens [2]. These paths to corrected electron microscopy are either to apply a fast time-varying field, to place a charge at the back focal plane or to use multipoles. Although, at this time, the resolution of electron microscopes was by far not yet limited by spherical or chromatic aberrations but by the not yet existing high stability of nowadays high resolution microscopes. Nevertheless, these first publications on theoretical electron optics and the following experimental set-up of correction systems stimulated the research in this field. The main goal of Scherzer, to improve the resolution by means of a correction system, could, however, not be achieved before he died in 1982. What could be demonstrated, as long as he was alive, was the proof of principle of the simultaneous compensation of the spherical and chromatic aberration of an objective lens [3].

The first Cs and Cc corrected electron microscope with which an improvement of resolution could be demonstrated was the development of a correction system for a low voltage scanning EM in 1995 [4]. Only two years later, in 1997, a breakthrough in high resolution TEM could be demonstrated by the successful completion of a Cs-corrected $200 \mathrm{kV}$ TEM [5] and of a dedicated STEM in 2003 [6]. It took another five to eight years before the first commercial Cs-corrected TEMs were available. Due to the easiness of the operation and the benefits of such a Cs corrected TEM - mainly the improved contrast and the vanishing delocalization of information - these instruments are widely accepted for high resolution and many systems have been installed worldwide.

After the success of Cs-correction the next step of the path towards ultra high resolution was the development of a correction system to compensate all resolution limiting aberrations of a TEM. These are not only the axial aberrations like the spherical and chromatic but also the compensation of the dominant off-axial aberration: the off-axial coma. A completely new correction system had to be developed. For this purpose an initiative was taken by M. Gibbson in the year 2000 and which was later taken over by U. Dahmen to form a team of several groups of DOE laboratories to set-up an electron microscope aiming for the unprecedented lateral resolution of $50 \mathrm{pm}$ for Material Science applications. The resolving power of this new unique instrument is not the only goal which had to be tackled. The required large field of view created made the compensation of the off-axial coma and the consideration of all coherent aberrations necessary. The ultimate aim is to achieve a so-called aberration-free imaging over the entire image. For this later purpose the acceptable phase-shift over the whole image due to residual coherent aberrations is just /4. This new TEAM (Transmission Electron Aberration-corrected Microscope) instrument is currently under development and almost ready for shipment to the National Center for EM in Berkeley. The most challenging part of this instrument is the new $\mathrm{Cc} / \mathrm{Cs}$-corrector with precise electron optical components and ultra stable power supplies.

The electron optical concept for the TEAM $\mathrm{C}_{\mathrm{C}} / \mathrm{C}_{\mathrm{S}}$-corrector is not as complex as the Ultracorrector proposed by $\mathrm{H}$. Rose [7]. The TEAM, as well as the Ultracorrector, corrector compensates Cs, Cc and the off-axial coma $\mathrm{B}_{3}$ of the objective lens and should lead to an aberration free imaging down 
to $50 \mathrm{pm}$ at $200 \mathrm{kV}$ and $300 \mathrm{kV}$ and a very large field of view. This large field of view fully exploits the capabilities of a $8 \mathrm{k} \times 8 \mathrm{k}$ CCD camera up to the information limit. This "Achroplanator", a $\mathrm{C}_{\mathrm{C}}, \mathrm{C}_{\mathrm{S}}$ and $\mathrm{B}_{3}$ compensated objective lens, is optimized for high resolution work between $80 \mathrm{kV}$ and $300 \mathrm{kV}$. It consists of 10 quadrupole-elements, a few Octupole-elements, several deflectors and round lenses to adapt the beam path according the theoretical concept and to allow the required precise alignment in order to achieve aberration free imaging up to a spatial frequency of $20 / \mathrm{nm}$. Due to this number of multipole elements we need an ultra stable power supply unit consisting of 151 current and 4 voltage supplies.

Currently this new C-COR has already proven its capability to compensate the spherical and chromatic aberrations and the off-axial coma. The resolution limiting incoherent disturbances, causing focus or image spread, are widely solved but not yet completely. So far the focus spread of the complete system - from the object plane down to the final image plane was estimated to be $0.34 \mathrm{~nm}$ at $300 \mathrm{kV}$ which is below the specification [8]. However, the image spread, caused by time varying dipole-fields is currently limiting the resolution to about $70 \mathrm{pm}$ at $300 \mathrm{kV}$. Within the coming weeks - before this new microscope will be transferred to Berkeley - the resolution limiting image spread has to be found and solved and the state of this experiment will be reported at this M\&M 2009 meeting.

" This work was conducted as part of the TEAM project funded by the Department of Energy, Office of Science" References:

[1] O. Scherzer, Z. Phys. 101 (1936) 593

[2] O. Scherzer, Optik 2 (1947) 593.

[3] H. Hely, Optik 60 (1982) 353.

[4] J. Zach, M. Haider, Nucl. Instr. Method A 363 (1995) 316.

[5] M. Haider, et.al. Nature 392 (1998) 768-769.

[6] O.L. Krivanek, et al., Ultramicroscopy 96 (2003) 229

[7] H. Rose Nucl. Instr. Meth. A 519 (2004) 12-27

[8] M. Haider, et al., Ultramicroscopy 108 (2008) 167

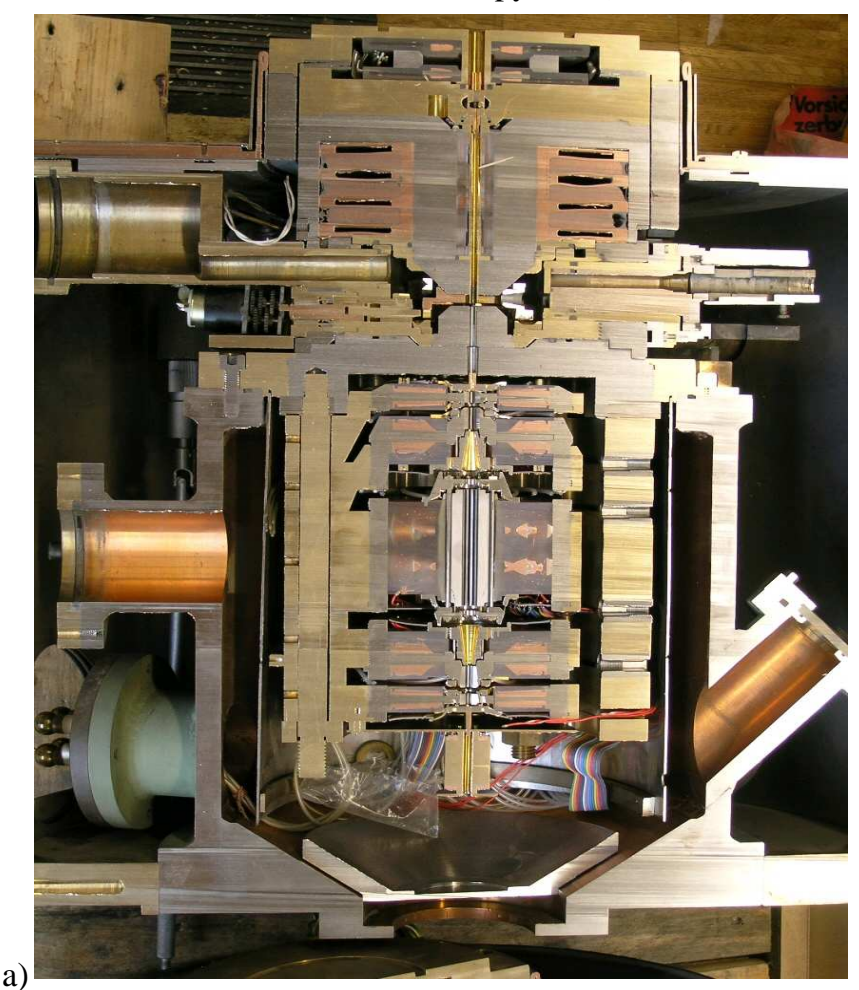

b)

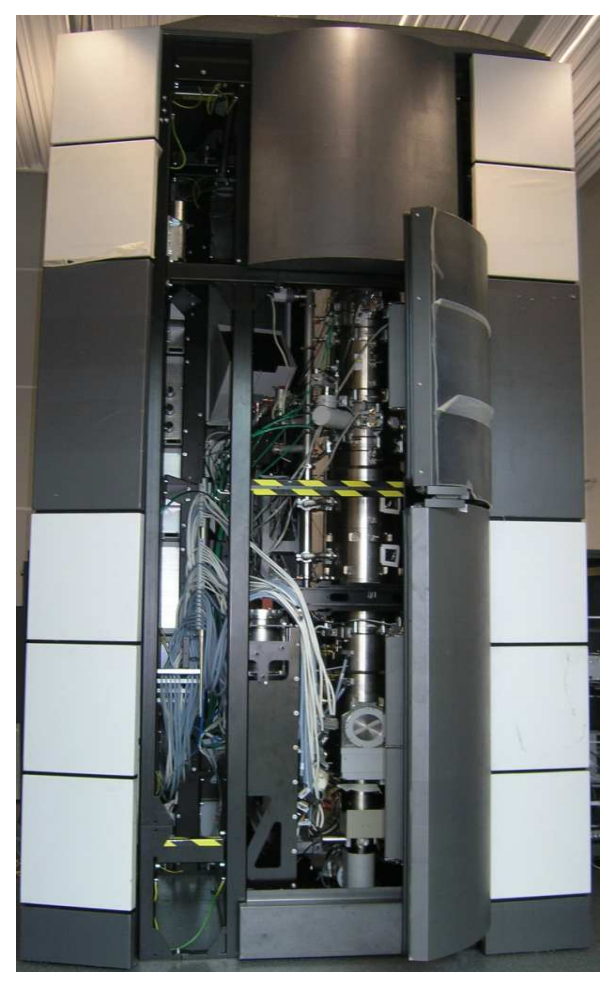

Fig. 1: a) The Cc/Cs-corrector of the Darmstadt project with which the proof of principle could be carried out and b) the Titan with the C-COR incorporated. The total height is more than $5 \mathrm{~m}$. 